\title{
Análisis descriptivos de procesos de remoción en masa en Bogotá
}

\author{
Descriptive analyses of landslide processes in Bogotá
}

Fecha de entrega: 17 de noviembre 2014 Fecha de aceptación: 1 de septiembre 2015

\section{Alfonso M. Ramos C. ${ }^{1}$, Mario Germán Trujillo-Vela ${ }^{1}$ y Luis Felipe Prada S. ${ }^{2}$}

\author{
${ }^{1}$ Instituto Geofísico, Pontificia Universidad Javeriana, Cra 7 No. 42-27. Bogotá, Colombia, a-ramos@javeriana.edu.co, \\ mario.trujillo@javeriana.edu.co \\ ${ }^{2}$ Departamento de Ingeniería Civil, Pontificia Universidad Javeriana, Cra 7 No 40-62, Bogotá, Colombia, \\ lf.pradas@javeriana.edu.co
}

Con el objetivo de tener mayores insumos para mejorar el entendimiento del problema de detonación de los procesos de remoción en masa y los factores que influyen sobre éstos, el presente articulo introduce una base de datos de procesos de remoción en masa para Bogotá, construida a partir de informes técnicos elaborados por el Instituto Distrital de Gestión del Riesgo y Cambio Climático IDIGER (antiguo FOPAE) tras la ocurrencia de cada evento de remoción de masa en la ciudad. Esta base de datos incluye 2208 eventos de movimientos en masa ocurridos en el perímetro urbano de Bogotá entre 1996 y 2013. Debido a las múltiples características reportadas en los informes, se recopilaron de forma sistemática variables como tipo de suelo, tipo de movimiento, posible causa detonante del evento, cobertura del suelo, pendiente promedio del lugar de la inestabilidad y el volumen de material deslizado, con el fin de condensar la mayor cantidad de información posible. Se exploró la relación entre el régimen de lluvias de Bogotá y la ocurrencia de movimientos en masa, encontrando una fuerte interrelación entre estas dos variables, marcado por un régimen bimodal con los máximos para los meses de abril-mayo y noviembre-diciembre. La serie histórica de movimientos en masa muestra una tendencia en la relación con el Índice Oceánico del Niño ONI, con algunas observaciones especiales para algunos años Niña en cuanto al incremento de ocurrencia de procesos de remoción en masa. Se elabora el autocorrelograma de la serie histórica de deslizamientos, con lo que se puede inferir que los eventos de remoción en masa están asociados a nivel temporal con la reactivación de procesos ocurridos 6 meses antes, lo que ratifica la relación lluvia deslizamiento de carácter bimodal.

Palabras clave: deslizamientos en Bogotá, análisis descriptivo de deslizamientos, correlación ONI-deslizamientos
In order to understand the processes that trigger landslides and the factors that may influence their generation such as geomorphology and climatology, this paper presents a new database of landslide processes compiled for Bogotá, constructed from technical reports elaborated by the Instituto Distrital de Gestión del Riesgo y Cambio Climático IDIGER (formerly known as FOPAE). This database includes 2208 landslide events that occurred between 1996 and 2013. Because of the many features described in the technical reports, it was necessary to categorize in a systematic way variables like soil type, type of movement, possible cause that triggered the event, ground cover, average inclination of the slope, and the volume of sliding material, in order to condense the largest possible amount of information. The database allows to infer relationships between precipitation and the occurrence of landslides in Bogotá. Analysis of the database shows a strong relationship between monthly rainfall and monthly amount of landslides, marked by a bimodal regime with the maximum recorded occurrence of slides between April-May and November-December. The time series of slides shows a hint of relationship with the Oceanic Niño Index ONI series, since for some Niña periods (more rain) the amount of recorded landslides increases. In addition, the correlogram of the time series of landslides is calculated, showing that the occurrence of landslide events may be associated with the reactivation of events that previously took place (six months in the past).

Keywords: landslides in Bogotá, descriptive analysis of landslide occurrence, correlation ONI-landslides 


\section{Introducción}

Los procesos de remoción en masa detonados por factores tanto naturales como antrópicos, causan daños importantes en lugares de altas pendientes. Las zonas montañosas tropicales son muy susceptibles a sufrir este tipo de problemas, debido a que generalmente se conjugan cuatro elementos fundamentales para la generación de fenómenos de remoción en masa: gradiente topográfico, sismicidad, meteorización de la roca parental y lluvias intensas (Suárez, 1998).

La lluvia es uno de los principales factores detonantes de deslizamientos. Sin embargo, definir la manera cómo influye en la estabilidad de los taludes no es una tarea fácil. Dicha dificultad se genera ya que el agua que entra en contacto con el talud a través de la precipitación interactúa con los materiales geológicos y puede disminuir sus propiedades mecánicas o puede aumentar las fuerzas de cuerpo que desestabilizan el talud. Una parte de los procesos de remoción en masa generados por lluvia se presentan debido al incremento en la presión de poros en el talud (o al aumento de la fuerza de infiltración específica) y las condiciones que favorecen o no la generación de procesos que están relacionados con la lluvia a través de la infiltración, características de los materiales geológicos, historia de la lluvia y geometría del talud (Wieczorek, 1996). La influencia de la lluvia sobre los procesos de remoción en masa depende fuertemente de las dimensiones y del tipo de deslizamiento. Se reconoce que los deslizamientos superficiales (flujos de lodos o flujos de detritos) son asociados a lluvias intensas de corta duración (Corominas y Moya, 1990; Aleotti, 2004; Coe et al., 2008), mientras que los deslizamientos profundos se relacionan principalmente con lluvias acumuladas en largos periodos (Bonnard y Noverraz, 2001; Dahal et al., 2006). Con base en lo anterior, se considera que no existe un único parámetro derivado de lluvia que sirva como determinador universal para la generación de cualquier tipo de proceso de remoción en masa.

Para tener mayor conocimiento sobre la generación de los procesos de remoción en masa y los factores detonantes, es importante hacer una adecuada recopilación de información de los deslizamientos producidos en un área determinada. Esto permite explorar la posibilidad de buscar relaciones entre algunas características geomorfológicas y climáticas con la ocurrencia de procesos de remoción en masa. Estas relaciones pueden surgir del análisis de regresiones entre series históricas de datos, de modelos basados en la física, de la experiencia de los expertos geotecnistas, de la formulación de modelos estocásticos o de observaciones de tipo experimental. Algunos trabajos basados en interpretaciones estadísticas de bases de datos, presentan ecuaciones de umbrales obtenidas al relacionar eventos de lluvia con deslizamientos. Estas ecuaciones de umbrales permiten decir si un evento de lluvia que supere ciertos niveles puede o no denotar movimientos en masa. Este tipo de relaciones se hace a partir de bases de datos de deslizamientos y registros de precipitaciones. Ejemplos de este tipo de aproximaciones se discuten en detalle en Glade (1998), Glade et al. (2000), Guzzetti et al. (2007, 2008), Dai y Lee (2001) y Li et al. (2011).

Una adecuada recopilación de información también permite alimentar y orientar el desarrollo de algunos modelos matemáticos basados en la física que describen la inestabilidad de taludes y la dinámica de movimientos en forma de flujos como los desarrollados por Iverson (2000), Iverson y Denlinger (2001), Pudasaini et al. (2005), Rickenmann et al. (2006), Wang et al. (2008), Luna et al. (2012) y Ouyang et al. (2013). Estas bases de datos también pueden ser procesadas con herramientas de minería de datos para encontrar la relación entre lluvia y deslizamiento (Pradhan et al., 2010). La importancia de contar con buena información de eventos de lluvia y de un inventario de deslizamientos motiva al desarrollo de una base de datos de deslizamientos que permita tener la información sistematizada y georreferenciada para avanzar en el conocimiento de la intrincada relación de los procesos de remoción con los eventos de lluvia. Este trabajo se realizó con base en el catálogo de eventos de remoción en masa registrados por el Instituto Distrital de Gestión del Riesgo y Cambio Climático IDIGER (antiguo FOPAE), entidad del gobierno local de la ciudad de Bogotá, durante el período de 1996 y 2013. La base de datos presentada en esta investigación se elaboró con el objeto inicial de realizar agrupaciones de eventos de remoción en masa en función de algunas características geométricas, geológicas y morfológicas para intentar relacionar estadísticamente las agrupaciones o tipologías de evento con una serie de variables derivadas de registros de precipitación en la zona de ocurrencia de los movimientos de remoción en masa. 
Dentro de los criterios para generar la agrupación de tipologías de eventos se consideraron variables como el tipo de material geológico, el tipo de movimiento registrado, la posible causa que detonó el evento, la cobertura del suelo, la pendiente promedio del lugar de la inestabilidad y el volumen de material deslizado. Las tendencias observadas, producto del análisis del catálogo de eventos de remoción en masa, se comparan con algunos resultados reportados en la literatura técnica. Se explora además la posible relación entre series históricas de precipitación registrada por algunas estaciones pluviométricas de Bogotá con los movimientos en masa registrados en un período de 17 años. Además se superpone la serie histórica del Índice Oceánico del Niño ONI para analizar la influencia de períodos de mayor o menor cantidad de lluvias con la ocurrencia de deslizamientos en la ciudad. Con esto se puede analizar si los eventos de remoción en masa presentados en la ciudad de Bogotá están relacionados con el régimen de lluvias presentadas en esta zona y si el comportamiento pudiese verse influenciado por los períodos con presencia del fenómeno de La Niña (temporada de lluvias por encima de los valores promedio en Colombia).

\section{Metodología para la construcción de la base datos de deslizamientos}

Con el fin de elaborar una base de datos con información relevante de los procesos de remoción en masa ocurridos en la ciudad de Bogotá, se revisó la información suministrada por IDIGER consistente en 7065 "diagnósticos técnicos", reportes oficiales donde se consigna la información detallada de la visita de expertos de IDIGER cuando ocurre un evento de remoción en masa, inundación o sismo en la ciudad. Esta base de datos abarca el período comprendido entre enero de 1996 y abril de 2013. La primera etapa del análisis de los diagnósticos técnicos consistió en filtrar la base de datos suministrada en función del tipo de riesgo (movimientos en masa) consignado en el inventario. Este primer proceso de filtrado redujo el número de diagnósticos técnicos de 7065 a 3776, los cuales fueron revisados de forma detallada, encontrando un número importante de reportes con dos o más eventos de remoción en masa. De esta manera se pudo recopilar información específica relacionada con 2208 eventos puntuales de remoción en masa (Figura 1). Los restantes 1652 informes no reportaban en realidad la ocurrencia de fenómenos de remoción en masa, sino que registraban visitas de diagnóstico a viviendas y obras de infraestructura con agrietamientos, o estudios detallados de amenaza por remoción en masa en algunas zonas de la ciudad.

Del total de eventos de remoción en masa encontrados en los informes (2208 eventos) se extrajo la información más relevante para poder generar una nueva base de datos con una estructura sistemática y compacta. Para cada evento se registró el número del diagnóstico técnico correspondiente (nomenclatura consecutiva empleada por el IDIGER), la fecha y hora de ocurrencia del deslizamiento, la ubicación de los procesos de remoción en masa (localidad, barrio o predio, dirección y/o coordenadas). Adicionalmente, se recopiló la información geotécnica relativa al tipo de movimiento, el volumen de material deslizado, la pendiente del terreno, la descripción geológica de la zona, el tipo de material deslizado, la posible causa del evento, el tipo de cobertura vegetal predominante en la zona y finalmente la fecha de emisión del diagnóstico técnico. Debido a las múltiples características de las descripciones geotécnicas de los procesos de remoción en masa, fue necesario agrupar en forma sistemática la información para poder analizarla posteriormente.

El tipo de movimiento se describió por medio de 13 categorías: avalancha, caída de roca, caída de bloques, deslizamiento, deslizamiento planar, deslizamiento rotacional, deslizamiento traslacional, erosión, flujo de lodo, flujo de material desagregado, flujo de roca, reptación y finalmente la categoría carente de esta información. La categoría deslizamiento se emplea como un término general para asociar los eventos en los cuales no era posible precisar, a partir de la información consignada en el diagnóstico técnico, el tipo de movimiento en masa generado o en donde el evento no fue clasificado explícitamente dentro de las restantes 12 categorías cuando se hizo la visita a campo y se produjo el consecuente diagnóstico técnico. Se incluyó una categoría adicional denominada combinación de tipo de movimiento la cual representa los movimientos de tipo complejo.

El tipo de material deslizado contempla tres categorías predominantes de geomateriales en los cuales se produjo el movimiento en masa: suelo, roca, detritos. Un alto porcentaje de los diagnósticos técnicos reportan los posibles factores detonantes de los eventos de remoción en masa. 
Esta información se registra en el campo "posibles causas" con las categorías: lluvia, excavación, explotación minera, rotura de tubería, sismo, intervención antrópica, expansión radicular, socavación, o no reporta causa. Finalmente, se extrajo la información concerniente al tipo de cobertura del suelo de la ladera donde ocurrió el evento, definiendo así las siguientes categorías: árboles, arbustos, gramíneas, sin capa vegetal, cubierta antrópica, combinación de diferentes coberturas, no registra tipo de cobertura. De los 2208 eventos de remoción en masa reportados en la base de datos, solamente 153 registran explícitamente la fecha de ocurrencia del evento, mientras que el resto cuenta con la fecha de visita a la zona por parte de los expertos del IDIGER. Esta visita pudo llevarse a cabo bien sea unas horas o hasta unos pocos días después de ocurrido el evento. Con el ánimo de corroborar las fechas presentadas en los informes, se planteó la posibilidad de complementar la información a través de bases de datos adicionales como los archivos de notas históricas en los periódicos locales y de grupos de investigación especializados en temas de riesgo.

Para la base de datos del diario "El Tiempo", el periódico de mayor circulación en Colombia, se utilizaron tres palabras clave en la búsqueda: deslizamientos, avalanchas y derrumbes. La información que pudo ser complementada corresponde a 7 eventos $(0.2 \%)$ para los cuales hubo coincidencia en la localidad, el barrio, la dirección de los predios afectados y una relación directa entre la fecha de elaboración del informe con la fecha de publicación de la noticia. Se revisó adicionalmente la base de datos de DesInventar, que recopila información desde 1994 bajo un marco conceptual y metodológico común por parte de grupos de investigadores, académicos y actores institucionales, agrupados en la Red de Estudios Sociales en Prevención de Desastres en América Latina (LA RED). Para esta búsqueda se realizó un filtrado por ubicación del lugar de ocurrencia de los eventos (Colombia, Bogotá D.C.), tipo de evento (deslizamientos, licuación, sedimentación y erosión), tipo de posibles causas (condiciones atmosféricas, desbordamiento, deslizamiento, erosión, inundación, La Niña, lluvias y sismo). La información encontrada aquí no permitió complementar la base de datos que se tenía debido a la ausencia de información precisa como la ubicación detallada de cada evento (ej. dirección o coordenadas) con las cuales se pudiera verificar que se trataba de un mismo evento reportado en los diagnósticos técnicos del IDIGER.

El compendio geográfico de los 2208 eventos procesados para la construcción de la base de datos se presenta en la Figura 1, donde se muestra la distribución espacial de los movimientos en masa reportados desde enero de 1996 hasta abril de 2013, para Bogotá. En el mapa se indican varias zonas separadas por líneas delgadas negras que representan la división administrativa de la ciudad en 20 localidades, con el fin de identificar aquellas localidades más afectadas por este fenómeno natural. Las localidades en las cuales se reporta la mayor ocurrencia de eventos de remoción en masa son: Ciudad Bolívar, San Cristóbal, Rafael Uribe y Usme, ubicadas en la zona periférica de la ciudad que se caracteriza por concentrar las zonas montañosas del área urbana, así como la población socioeconómica más vulnerable de la ciudad. En la Figura 1 también se presentan nueve estaciones meteorológicas con registros diarios u horarios de lluvia (triángulos), que se utilizaron en esta investigación para hacer el análisis de la relación entre los registros de precipitaciones con la serie histórica de movimientos en masa. Las franjas de colores representan las zonas climáticas de la ciudad según la metodología de Thornthwaite empleada por IDEAM (2005). Vale la pena anotar que en la localidad de Ciudad Bolívar, donde se registran mayor número de eventos de remoción de masa en el período de análisis considerado, la clasificación climática corresponde a la categoría de clima semi-seco con un régimen de precipitación promedio anual entre $600 \mathrm{~mm}$ y $700 \mathrm{~mm}$, mientras que en las zonas con mayor precipitación (ubicadas al norte de Bogotá) se pueden tener registros anuales entre 1200 y $1400 \mathrm{~mm}$. La Figura 2 describe la ubicación de los deslizamientos recopilados en esta investigación, clasificados según el tipo de geomaterial en el cual se presentó el movimiento, de acuerdo con la información reportada en los diagnósticos técnicos (suelo, roca, detritos, sin descripción). La mayor parte de los deslizamientos ocurrió en suelos, según la información recopilada.

\section{Análisis de la información de deslizamien- tos y lluvia}

En esta sección se describe el proceso de consolidación de la información existente de los 2208 eventos en función de la calidad de los datos consignados en los reportes. Los criterios de clasificación se basaron en la existencia o no 


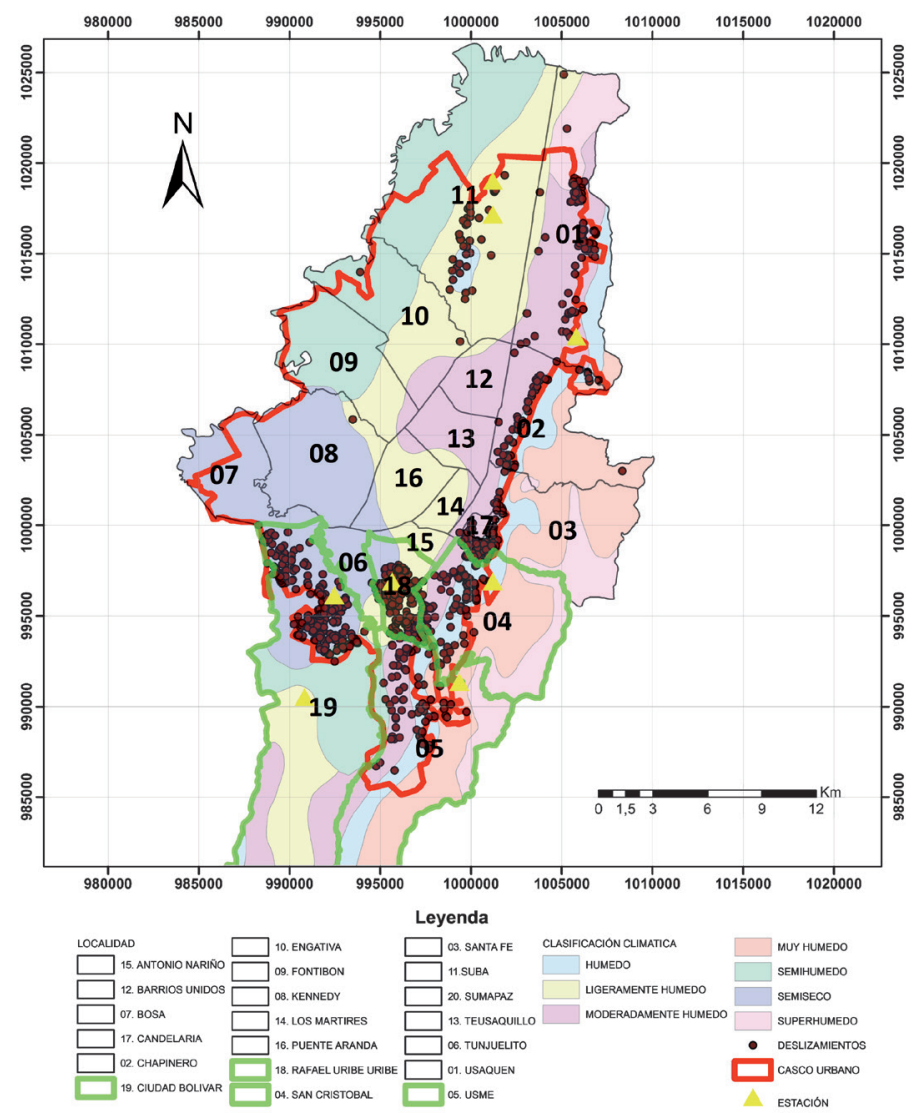

Figura 1: Mapa de Bogotá con los eventos de remoción en masa reportados desde 1996 a 2013. La localidad de Ciudad Bolívar (costado sur occidental de la ciudad) es la zona con mayor número de reportes de deslizamientos y corresponde a una zona climática semi-seca según la escala de Thornthwaite.

de datos fundamentales para el análisis de relación lluviadeslizamiento tales como la fecha de ocurrencia del evento y la ubicación del mismo. En la Figura 3a se resume el inventario de los 3882 eventos analizados inicialmente, en donde se encontró que solamente 153 procesos de remoción en masa ( $4 \%$ del total de los consultados) contaban con la fecha y dirección de ocurrencia del evento, mientras que 1968 eventos (equivalentes al 51\%) no registran la fecha de ocurrencia del evento. El restante $45 \%$ corresponde a otro tipo de fenómeno natural registrado en el informe (categorizado como No aplica) diferente a procesos de remoción en masa, junto con otros casos de informes que no registran fecha y dirección, o informes que aparecían en los listados de inventarios pero que no fueron encontrados en los archivos (No existe). En la Figura 3b se agrupan los procesos de remoción en masa en función de las localidades de Bogotá en donde se reportó cada uno de los eventos. La mayoría de los movimientos se han presentado en la

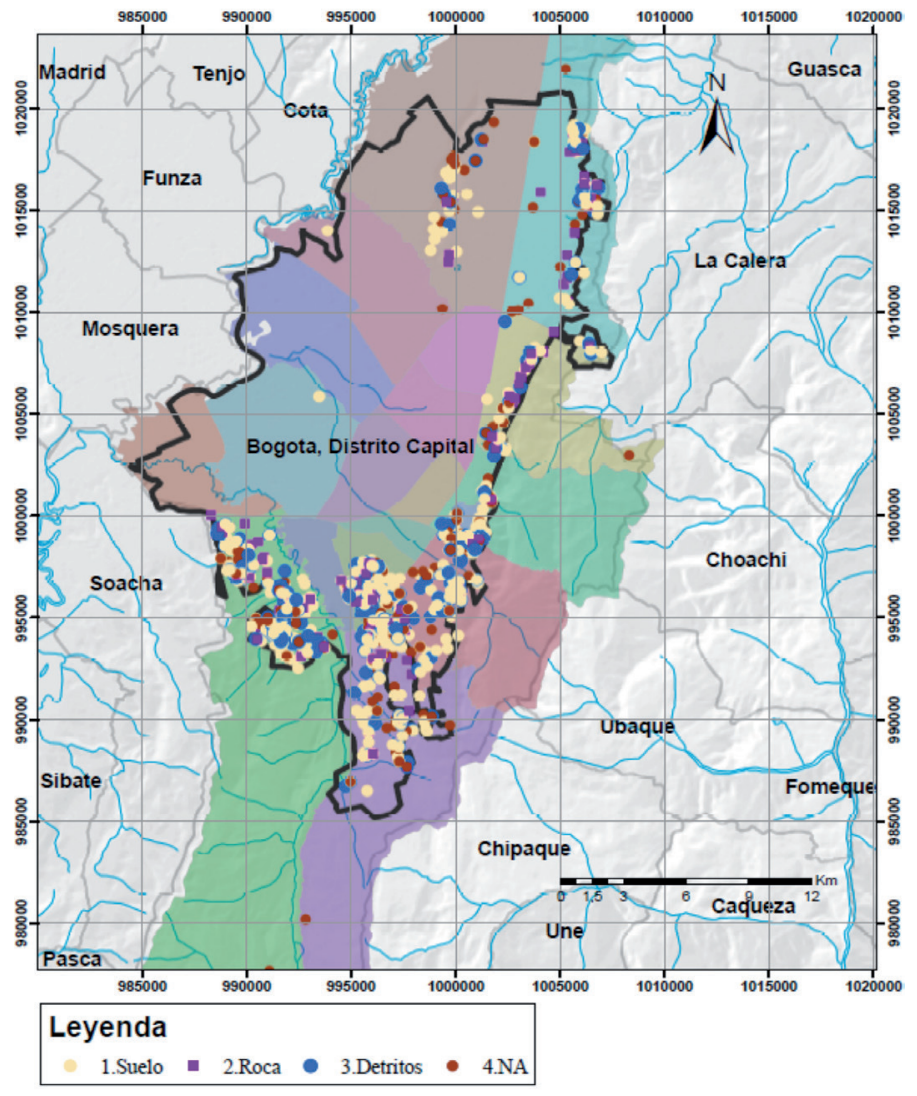

Figura 2: Mapa de Bogotá con los eventos de remoción en masa reportados desde 1996 a 2013, agrupados en función del tipo de geomaterial (suelo, roca, detritos, sin información) en el cual se reportó el proceso de inestabilidad geotécnica.

localidad de Ciudad Bolívar con un 30.21\%, seguida por las localidades de San Cristóbal con 17.35\%, Rafael Uribe con $16.08 \%$, Usme con $11.10 \%$ y Santa Fe y Usaquén, ambas con $7.56 \%$. Estas localidades cubren gran parte de la zona de piedemonte de los cerros orientales y las áreas de topografía ondulada en terrenos formados por rocas terciarias y depósitos de ladera hacia el sur. En algunas partes se observan afloramientos de la formación Guaduas constituida por arcillolitas rojizas y abigarradas, mantos de carbón, areniscas y arcillolitas grises depositadas en un ambiente de transición marino-continental hacia finales del cretáceo y comienzos del terciario (Moya y Rodríguez, 1987). Otras localidades presentan un porcentaje inferior al $6 \%$ de ocurrencia de eventos de remoción en masa: Chapinero, Suba, Sumapaz, Candelaria, Engativá, Puente Aranda, Fontibón y Teusaquillo. En las localidades de Antonio Nariño, Barrios Unidos, Bosa, Kennedy, Mártires y Tunjuelo no se reportaron fenómenos de remoción 
en masa. Como es de esperarse, la geomorfología predominante en estas últimas localidades corresponde a zonas planas y valles aluviales.
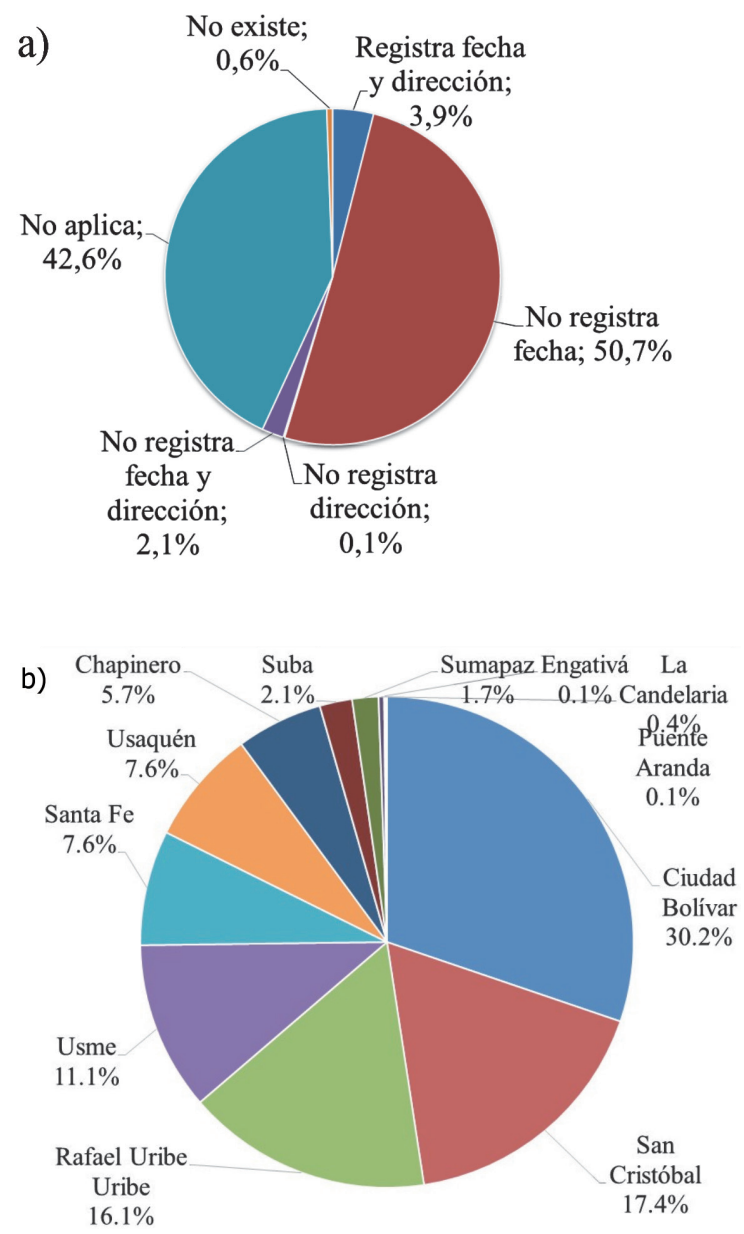

Figura 3: Estadísticas obtenidas del catálogo de eventos de remoción en masa, a) descripción de información básica reportada (fecha y ubicación) y b) porcentaje de movimientos en masa registrados por localidad.

La Figura 4a representa la estadística del tipo de material geológico predominante del talud en donde se presentó el proceso de remoción en masa, de acuerdo con la información consignada en los diagnósticos técnicos elaborados por los funcionarios del IDIGER. Se observa que un $52 \%$ de los diagnósticos técnicos establecen que el tipo de material deslizado corresponde a suelo, seguido de un $22 \%$ que no establece el tipo de material deslizado, un $13 \%$ de material tipo roca y $13 \%$ para detritos. De una forma semejante Guzzetti et al. (2008) presentan también algunas estadísticas realizadas para las bases de datos de lluvias que generan deslizamientos, donde el desconocimiento de la litología para donde ocurrió el evento tiene un porcentaje del 36.4\%. En la Figura $4 \mathrm{~b}$ se agrupan los procesos de remoción en masa dependiendo de la clasificación del movimiento registrada por los especialistas del IDIGER en los diagnósticos técnicos. El $36 \%$ de los eventos registrados fueron clasificados como deslizamientos, indicando que no hay especificidades en el movimiento ocurrido que permitan asociarlo con las demás categorías. El 38\% de los movimientos que se presentan en Bogotá ocurren en forma de flujos de material desagregado y de lodos, el 13\% se refiere a los movimientos donde se observan superficies de corte (deslizamiento rotacional, traslacional y planar), el $10 \%$ clasifican como caídas y un $4 \%$ se asocia a fenómenos de erosión y reptación. Los movimientos en forma de flujos son los más frecuentes en la ciudad, lo cual concuerda con las tendencias observadas por otros investigadores en diferentes países. Un ejemplo de esto es un estudio generado por Guzzetti et al. (2008), que reporta el $42 \%$ de participación para los flujos de detritos del total de fenómenos de remoción de masa registrados en una base de datos global de 2626 eventos detonados por lluvia en el período comprendido entre 1917 y 2005 . De igual manera el $42 \%$ de los desastres causados por movimientos en masa en Japón entre los años 1989 y 2007, son atribuidos a los flujos de detritos (Shrestha et al., 2008). Los flujos de detritos representan el $32 \%$ de 252 casos de movimientos en masa de la cuenca vertiente al embalse de Rules en la provincia de Granada, España (Jiménez-Perálvarez et al., 2010).

La Figura 4c muestra los procesos de remoción en masa en función de las posibles causas que generaron el evento. Estas posibles causas surgen de la evaluación y criterio de los expertos del IDIGER, tal como quedó consignado en los diagnósticos técnicos. Para el 39\% de los eventos documentados, la posible causa del movimiento fue la 1luvia. Para el $8 \%$ de los eventos se determinó que la posible causa fue la intervención antrópica y en proporciones inferiores al 3\% se asoció la ocurrencia de los deslizamientos con sismos, excavaciones, explotación minera y rotura de tuberías. Esta estadística permite afirmar que la lluvia es el factor detonante más importante en los eventos de remoción en masa en el perímetro urbano de la ciudad. Esto puede ser verificado en las Figuras 7 y 8 donde se puede observar que hay dos períodos en el año 
a)

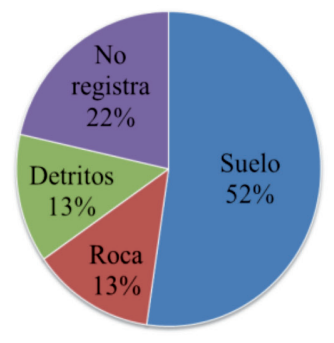

c)

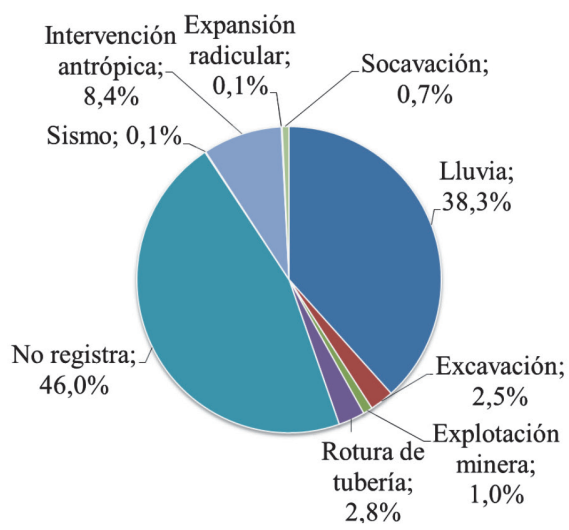

e)

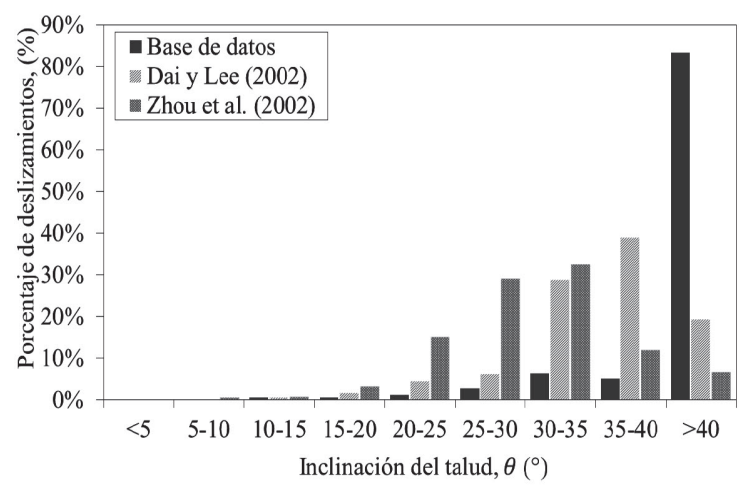

b)
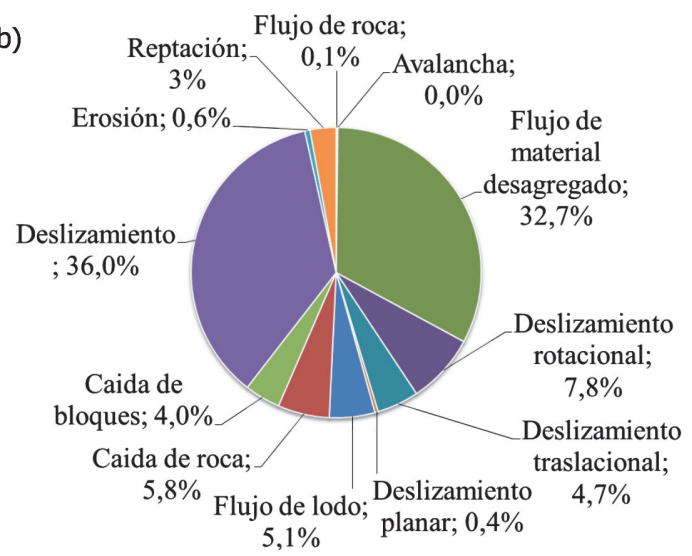

d)

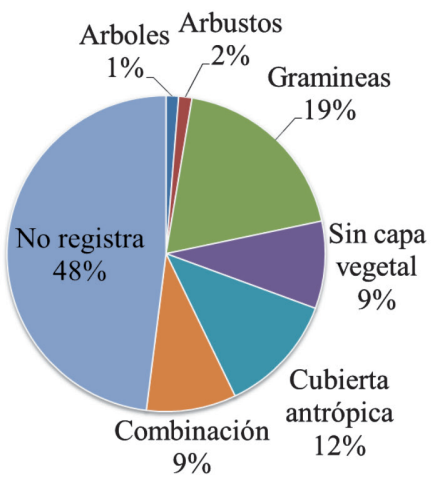

f)

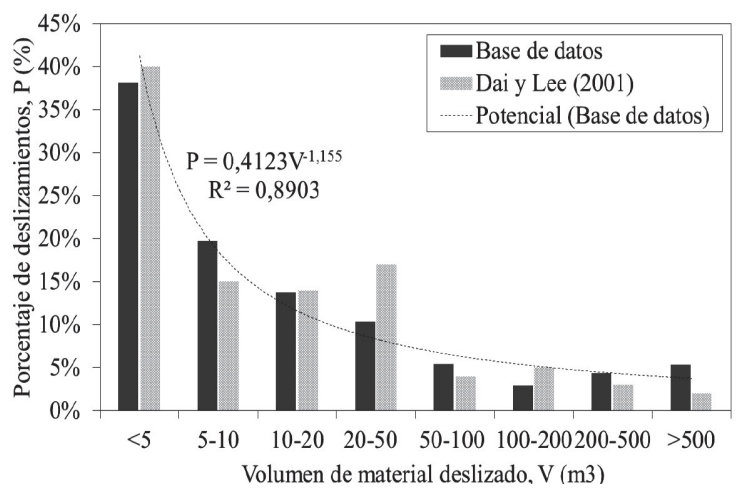

Figura 4: Estadísticas del catálogo de eventos de remoción en masa. a) Descripción general del tipo de material deslizado, b) tipo de procesos de remoción en masa generados en Bogotá, c) posible causa que desencadenó el movimiento en masa, d) tipo de cobertura que predomina en la zona del movimiento, e) ángulos de inclinación del talud en función de la frecuencia relativa de eventos de remoción en masa y f) volumen de material deslizado en función de la frecuencia relativa de eventos de remoción en masa.

en las que tanto las precipitaciones como la cantidad de deslizamientos generados aumentan de manera importante. El porcentaje que corresponde a la intervención antrópica (8\%) es el segundo más importante, el cual quedará soportado por las inferencias presentadas más adelante en esta misma sección.

En la Figura 4d se presenta como criterio de clasificación el tipo de cobertura superficial que fue registrada en el lugar de ocurrencia del proceso de remoción en masa.
Es importante contrastar dos estadísticas obtenidas de los tipos de coberturas: la primera, es el porcentaje de procesos de remoción en masa correspondientes a las coberturas antrópicas $(12 \%)$. Este tipo de cobertura está asociado en estas zonas a obras de autoconstrucción carentes muchas veces de diseños y procesos constructivos adecuados. También es frecuente la ausencia de obras de estabilización de laderas previas a la construcción de las viviendas. Este tipo de intervenciones aumentan el grado de 
susceptibilidad de los taludes, lo cual puede desencadenar fenómenos de remoción en masa durante la temporada de lluvias. El segundo tipo de cobertura que más se registra en los procesos de remoción en masa es la vegetación (plantas gramíneas, arbustos, árboles) que representan el $22 \%$ de los registros. Sin embargo, es importante aclarar que las coberturas dominadas por árboles tan solo representan el $1 \%$ de los procesos de remoción en masa analizados. A partir de esta estadística, al igual que las reportadas en Zhou et al. (2002), se puede evidenciar que cuando el suelo está protegido con árboles se registra una menor cantidad de deslizamientos. Algunos trabajos han mostrado que una cobertura vegetal, de tipo leñoso, con un sistema de raíces fuertes, grandes y profundas ayuda a mejorar la estabilidad de las laderas (Gray y Leiser, 1982; Greenway, 1987; Imaizumi et al., 2008), aunque se necesitan más estudios para comprender mejor el papel de la cobertura vegetal en la ocurrencia de deslizamientos de tierra (Zhou et al., 2002). Esto permitiría inferir que un porcentaje importante de los eventos de remoción en masa que se presentan en Bogotá, se pueden atribuir no solo a la influencia de las intervenciones antrópicas de bajo nivel técnico, sino también a las lluvias antecedentes que saturan el suelo gradualmente (más que a eventos de lluvias cortas con grandes intensidades).

La Figura 4e presenta la estadística de cantidad de deslizamientos ocurridos en función de los ángulos de inclinación de los taludes (medido con respecto a la horizontal). La información de inclinación de las laderas se obtuvo del procesamiento de un modelo digital de elevación, generado a partir de topografía de detalle con curvas de nivel espaciadas un metro, el cual se superpuso con la localización geográfica de los eventos de remoción en masa. La mayor cantidad de procesos de remoción en masa se presentan para laderas con ángulos de inclinación mayores a $40^{\circ}$. Para ángulos menores de $40^{\circ}$, la frecuencia es inferior al 6\% (Figura 4e). Esto podría dar cuenta de lo importante que resulta la intervención antrópica en laderas de alta pendiente para la construcción de viviendas carentes de diseño estructural ceñido a la normativa nacional, con deficiente control de materiales y del proceso constructivo y sin la construcción de obras geotécnicas adecuadas para estabilizar las laderas, tal como se puede apreciar en la Figura 5. Imaizumi et al. (2008) reportan un comportamiento semejante al observado en esta investigación, donde la mayor frecuencia de ocurrencia de deslizamientos por cada $\mathrm{km}^{2} / \mathrm{año}$, se presenta en laderas con pendientes mayores a $30^{\circ}$, cubiertas de bosques jóvenes con edades entre 1 y 10 años. Otras investigaciones han encontrado relaciones directas entre mayores frecuencias de generación de procesos de remoción en masa con altas inclinaciones de las laderas, pero con rangos de pendientes diferentes al observado en Bogotá, como lo reporta Dai y Lee (2002) para Hong Kong, donde la mayor cantidad de deslizamientos se registró para taludes entre $30^{\circ}$ y $40^{\circ}$ de inclinación. Zhou et al. (2002) muestran una mayor frecuencia de deslizamientos en taludes con ángulos de inclinación entre $20^{\circ}$ y $35^{\circ}$ para la isla de Lantau en Hong Kong.

La Figura 4f muestra la cantidad de eventos de remoción en masa en función de la magnitud del proceso, expresado en términos del volumen de material deslizado. Los registros procesados en esta investigación indican que el $82 \%$ de los movimientos en masa acarrean volúmenes de material inferiores a $50 \mathrm{~m}^{3}$. El restante $18 \%$ son procesos de remoción que tienen volúmenes mayores a $50 \mathrm{~m}^{3}$, incluyendo un $1 \%$ de los movimientos que reportan volúmenes mayores a $1000 \mathrm{~m}^{3}$ (Figura 4f). Moya Sánchez et al. (2013) indican que la mayor cantidad de desprendimientos de bloques en la ladera Solà d'Andorra en Andorra la Vella, Principado de Andorra, desplazan volúmenes entre los 4 y los $30 \mathrm{~m}^{3}$, alcanzando el mayor los $150 \mathrm{~m}^{3}$. Dai y Lee (2001) reportan que el volumen de las fallas va desde 0.1 hasta $10^{5} \mathrm{~m}^{3}$ y la representación del porcentaje de deslizamientos en función del volumen también está regida de forma semejante con una ley de potencia al igual que Brardinoni y Church (2004). Estas cifras demuestran que la proporción más alta de eventos de remoción en masa transportan volúmenes pequeños de material, tal como se puede ver en la Figura 4f, y que la relación se puede describir por medio de una ecuación de decrecimiento exponencial.

La Figura 6 caracteriza la serie histórica mensual de eventos de remoción en masa. Es notorio el incremento de eventos de remoción en masa registrados en los años 2010 y 2011 de la serie de tiempo, período que coincide con la ola invernal asociada con el fenómeno de La Niña que más daños ha causado en Colombia en las últimas décadas. En estos años el incremento es tan importante, que el número de deslizamientos mensuales excede el valor de 
una desviación estándar. La tendencia observada puede atribuirse a una intensificación de los períodos invernales en la última década, sumado al crecimiento del casco urbano de la ciudad que interfiere con el equilibrio natural de los geosistemas al modificar por ejemplo la capacidad de infiltración y almacenamiento del suelo.

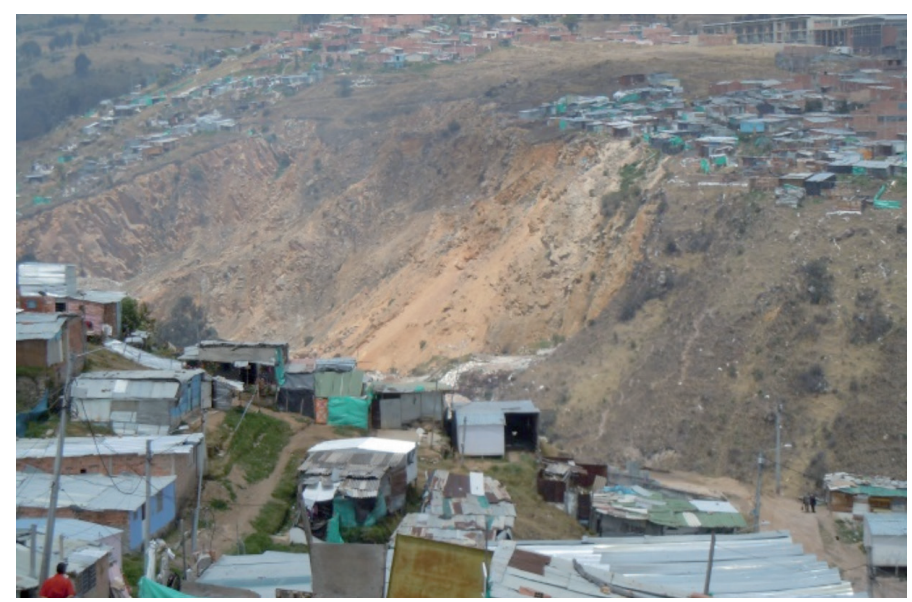

Figura 5: Fotografía de un proceso de remoción en masa del tipo flujo de detritos en un sector del sur de Bogotá, en la localidad de Ciudad Bolívar.

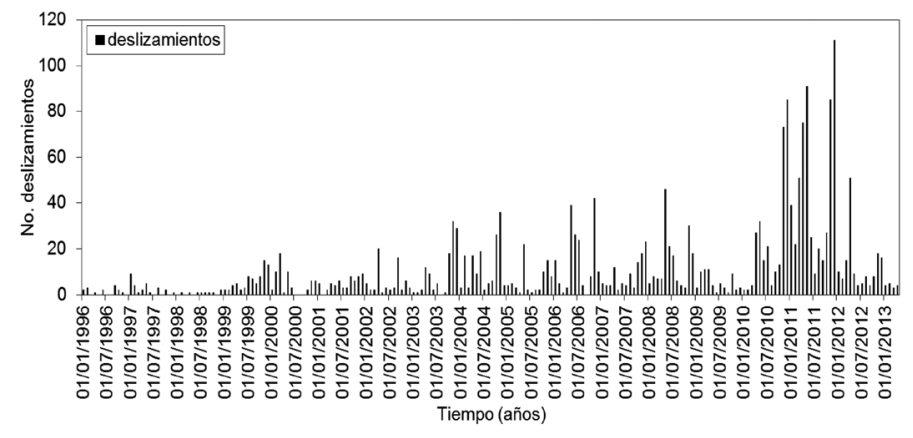

Figura 6: Serie histórica de deslizamientos de 17 años en Bogotá, período 1996-2013.

Es importante conocer la dependencia que tiene una variable consigo misma en un período de registro, es decir, que tanto depende un valor determinado de los valores anteriores. Esto se puede medir a partir de la función de autocorrelación, tal como se presenta en los autocorrelogramas de la Figura 7. En la Figura 7a se puede ver el autocorrelograma y en la Figura $7 \mathrm{~b}$ el autocorrelograma parcial calculados a partir de la cantidad mensual de eventos de remoción en masa registrados en los 17 años de información disponible. El autocorrelograma simple (Figura 7a) brinda para un retardo $k$ la correlación entre los valores de deslizamientos observados con una diferencia de $k$ meses, como la correlación para retardos menores, mientras que el correlograma parcial (Figura 7b) brinda la correlación estricta entre el dato analizado y el rezago deseado a $k$ meses.
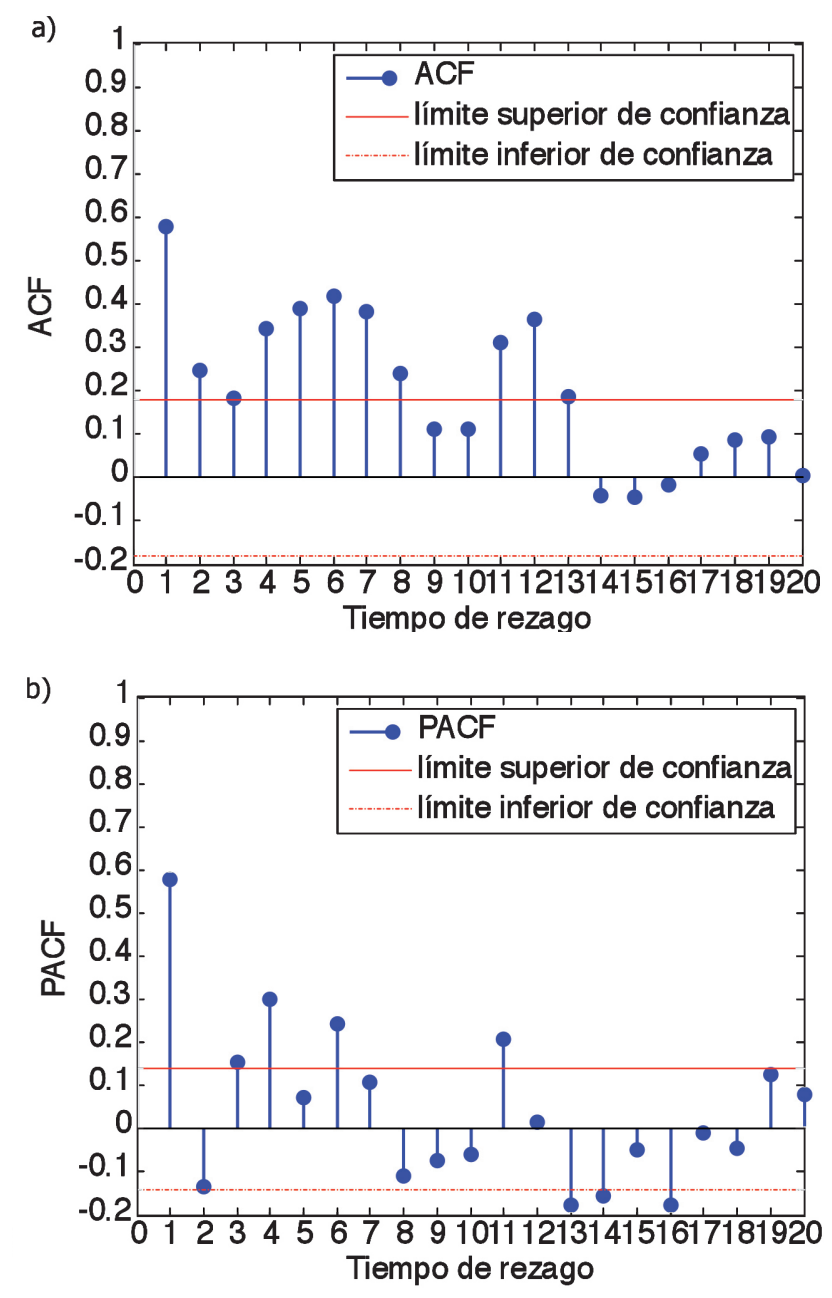

Figura 7: a) Función de autocorrelación para la serie de tiempo de los eventos mensuales de remoción en masa y b) función de autocorrelación parcial para la serie de tiempo de los eventos mensuales de remoción en masa.

De la Figura 7 se puede concluir que la serie histórica tiene una memoria larga que disminuye en forma no monotónica hacia valores de autocorrelación nula o poco significante (espacio comprendido entre las líneas rojas). Se puede ver también que la serie temporal está influenciada por un período de retardo de 6 y 12 meses. Esto podría interpretarse como si un porcentaje importante de los eventos que ocurren hoy en día pudieran ser en realidad la reactivación de eventos inminentes u ocurridos hace 6 o 12 meses, lo cual se podría asociar también al período del régimen bimodal de precipitación característico de la ciudad de Bogotá (Figura 8). En el autocorrelograma 
parcial se nota una correlación significativa de los eventos de remoción en masa con los eventos que acontecen 1 mes antes, o 4 o 6 meses antes.

La relación de los eventos de remoción en masa con el régimen de lluvia de Bogotá se puede analizar a partir de los resultados de la Figura 8. En los períodos abril-mayo y noviembre-diciembre tanto las máximas precipitaciones como la mayor cantidad de movimientos en masa coinciden en estos meses del año. La Figura 8 muestra la agrupación de eventos de remoción en masa expresada como la serie de promedios mensuales multianuales ocurridos en los 17 años en referencia (barras grises). A las estadísticas mensuales multianuales se le superpuso el valor correspondiente de precipitación media mensual multianual del mismo período de análisis, registrado en 9 estaciones pluviométricas que cuentan con buena longitud y calidad de datos, ubicadas en 9 localidades diferentes donde se registra el mayor número de fenómenos de remoción en masa (líneas continuas y discontinuas). Las series de deslizamientos tienen un rezago aproximado de un mes respecto a la precipitación media mensual multianual, en donde la mayor cantidad de movimientos en masa se presenta tiempo después de la ocurrencia de los valores más altos de precipitación en la ciudad (Figura 8). También se observa que esta tendencia general no se replica en las estaciones de San Cristóbal y Usme (líneas discontinuas), en donde el régimen hidrológico difiere del resto de la ciudad al presentarse los mayores valores promedio mensuales de lluvia en el período entre juniojulio-agosto y noviembre. Estas discrepancias indican la necesidad de hacer análisis zonificados en la ciudad para relacionar de forma más precisa los eventos de lluvia con los eventos de remoción en masa, dada la alta variabilidad espacial de la precipitación en el área. La tendencia que se evidencia en Bogotá es diferente a la que se presenta en otros lugares con estacionalidad como Hong Kong, en donde el régimen de lluvias es monomodal, y el promedio de deslizamientos por mes sigue la misma tendencia del régimen meteorológico de la zona como lo muestra Chau et al. (2004).

Comúnmente los períodos extremos de lluvias y sequías son relacionados al índice macroclimático ONI (Oceanic Niño Index). El ONI es una medida de la variación de la temperatura superficial del océano en períodos de 3 meses con respecto a los valores promedio históricos (de una serie base entre enero de 1950 y diciembre de 1999) en una región particular (zona 3.4-Niño delimitada con las coordenadas $120^{\circ} \mathrm{W}-170^{\circ} \mathrm{W}, 5^{\circ} \mathrm{S}-5^{\circ} \mathrm{N}$ ) donde se monitorea permanentemente el fenómeno del Niño (NOAA, 2014). Valores negativos del ONI indican la ocurrencia de un fenómeno Niña, el cual dependiendo de su intensidad puede traer volúmenes de precipitación por encima de los valores promedio observados para las regiones Caribe y Andinas de Colombia.

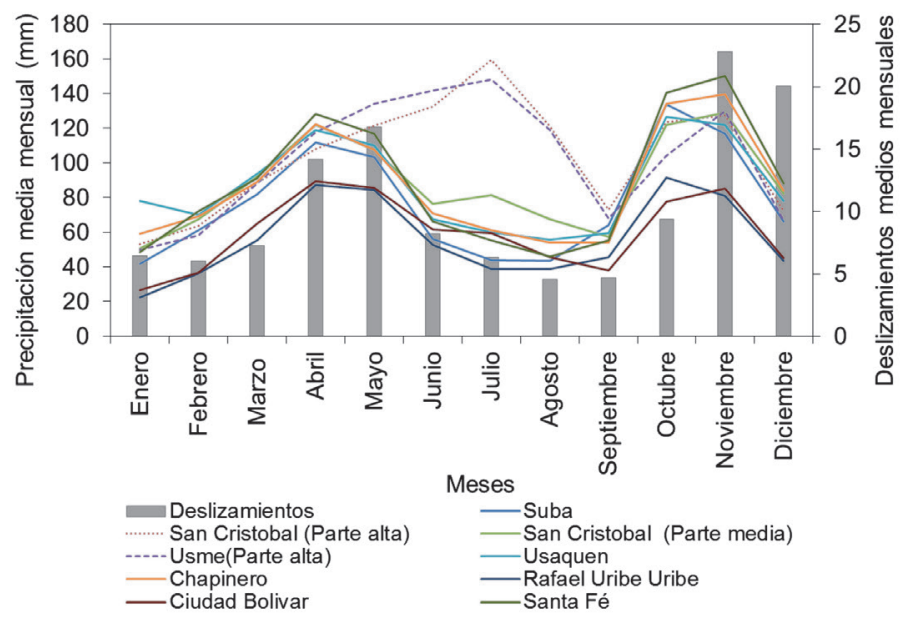

Figura 8: Precipitación media mensual multianual para 9 localidades de Bogotá y deslizamientos promedio mensuales multianuales.

Debido a la relación que se encontró entre el régimen de lluvias y el régimen de ocurrencia de procesos de remoción en masa para Bogotá, es importante determinar si existe alguna relación entre los registros del ONI y la serie histórica de movimientos en masa. Para ello se superpuso la serie histórica del ONI tomado de la NOAA (2014) y la serie histórica de movimientos en masa entre los años 1996 y 2013 (Figura 9). En la Figura 9 se puede apreciar algún tipo de relación entre los periodos de lluvia en Bogotá (La Niña) y la mayor cantidad de deslizamientos mediante las ventanas subrayadas horizontalmente de color azul, entre los años 1996-1997, 1998-2001, 2007-2008 y especialmente los años 2010-2012, en donde la cantidad de deslizamientos se superpone con el índice marcado como Niña. También las ventanas subrayadas diagonalmente de color amarillo, entre los años 1997-1998, 2002-2003 y 2009-2010, son tiempos en donde se presentan menos deslizamientos ya que estos periodos corresponden a la época de Niño o de sequía para Bogotá. 


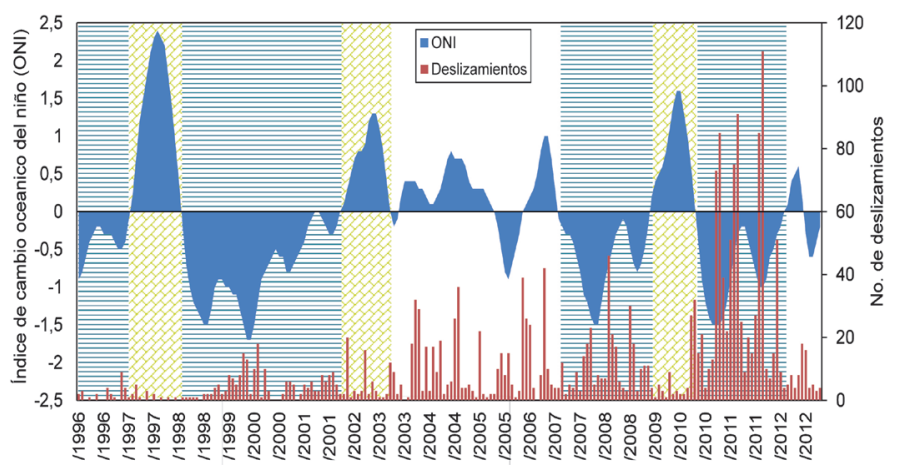

Figura 9: Evolución del índice macroclimático ONI con la ocurrencia de eventos de remoción en masa en Bogotá

A diferencia de los resultados presentado en la Figura 9, un análisis estadístico hecho para los Andes Centrales en la provincia de Mendoza, Argentina, indica que se registró una mayor actividad de deslizamientos durante la fase ENSO El Niño en la Cordillera Frontal. Sin embargo, esto no ha sucedido en la precordillera. Por otra parte, durante la fase fría (La Niña), los movimientos de taludes en particular disminuyeron en la Cordillera Frontal, con cantidades similares a las de la Precordillera (Moreiras, 2005). Esto indica lo importante y marcada que es la influencia de variación meteorológica en la generación de procesos de remoción en masa en diferentes zonas de América, en función del Índice Oceánico del Niño, tal como lo describe Trauth et al. (2003). Además la variación climática se puede marcar en áreas mucho más pequeñas como sucede en Bogotá tal como se muestra en la Figura 8, lo que corrobora la importancia de generar relaciones lluvia-deslizamientos con la mejor resolución y discretización espacial posible.

\section{Conclusiones}

La mayoría de los movimientos en masa se registraron en las localidades que tienen zonas de altas pendientes como Ciudad Bolívar, San Cristóbal, Rafael Uribe, Usme, Santa Fe y Usaquén. Estos eventos se han asociado tanto a la precipitación acumulada como a la intervención antrópica, representada en la ejecución de excavaciones, rellenos y autoconstrucción de viviendas carentes de diseños técnicos adecuados que aumentan la susceptibilidad de las laderas. El tipo de movimiento en masa más reportado en la base de datos elaborada en esta investigación es el flujo de materiales desagregados con una participación del 33\%. La categoría de deslizamientos (puede abarcar diferentes clases de procesos de remoción en masa) tiene una contribución del $36 \%$. Este alto porcentaje se puede explicar a partir de la amplia definición de dicha categoría, teniendo en cuenta que ésta se usa para asociar los eventos en los cuales no se cuenta con suficiente información para poder clasificar específicamente el fenómeno en otra clase de movimiento en masa. El tipo de material deslizado que más se reporta corresponde a suelo y detritos, siendo esto congruente con los tipos de movimiento más registrados (flujos y deslizamientos).

Por otro lado, según el análisis de la base de datos creada en esta investigación, el factorque puede tenermayor influencia en la generación de los procesos de remoción en masa para Bogotá es la lluvia acumulada. Se recomienda continuar el análisis de la base de datos descrita en este documento, con el fin de poder generar umbrales de lluvia asociados a deslizamientos. Esto permitirá a futuro implementar sistemas de alerta temprana en la ciudad a partir del monitoreo de las variables relevantes de lluvia, definidas en función del régimen hidrológico y de las condiciones geomorfológicas particulares de cada sector de la ciudad. Dentro de los factores que influyen en la estabilidad de taludes, es importante destacar que la cobertura del suelo juega un papel importante en el aumento de la resistencia del suelo. Los análisis descriptivos presentados en este trabajo pueden ser corroborados con otros autores que destacan la significancia de la vegetación de tipo leñosa para incrementar la resistencia de las laderas. De igual manera, se puede concluir que las laderas cubiertas con árboles es donde menos se presentan deslizamientos, a diferencia de suelos cubiertos por arbustos y gramíneas.

Se encontró que para Bogotá, la frecuencia de deslizamientos en función del ángulo de inclinación del talud puede ajustarse con una ley de crecimiento exponencial, en donde la mayor cantidad de eventos se presentan en pendientes por encima de los $40^{\circ}$. Esto se podría asociar a fenómenos de caída de bloques, volcamientos y flujos de material desagregado registrados en las zonas de la ciudad donde también es evidente la intervención antrópica que desencadena el aumento de la susceptibilidad de las laderas. Por otro lado, se logró encontrar una relación entre la frecuencia de deslizamientos y el volumen de material deslizado. Dicha relación se puede representar por medio de una ecuación 
de decremento exponencial, donde la mayor frecuencia de movimientos se presenta para volúmenes inferiores a 50 $\mathrm{m}^{3}$.

Finalmente, el autocorrelograma de la serie histórica de deslizamientos muestra que los registros tienen una memoria larga que disminuye en forma monotónica hacia valores de autocorrelación nula o poco significante. Se puede observar que la serie temporal se ve influenciada por un período de retardo de 6 y 12 meses, lo cual podría estar asociado al régimen bimodal de precipitación característico de la ciudad de Bogotá. Esta característica se puede corroborar al superponer la serie del Índice Oceánico del Niño ONI con la serie histórica de deslizamientos, en donde se encontró de forma cualitativa, una relación entre los máximos registros de deslizamientos y las épocas de Niña (periodos de lluvia para Bogotá), tal como se registró en la última oleada invernal de 2010 y 2011 en la ciudad.

\section{Agradecimientos}

Los autores agradecen a la Pontificia Universidad Javeriana por el soporte para el desarrollo del presente trabajo bajo proyecto de investigación No: 6143-Determinación de niveles umbrales de lluvia que generan deslizamiento. También desean agradecer la colaboración del IDIGER al permitir consultar y usar la base de datos de los diagnósticos técnicos de eventos de remoción de masa ocurridos en Bogotá entre 1996 y 2013. Agradecemos también a Edwin Castillo del IDIGER por el apoyo institucional, y a Carolina Urquijo y Ana Carolina Santos por la generación de los mapas mostrados en el estudio.

\section{Referencias}

Aleotti, P. (2004). A warning system for rainfall-induced shallow failures. Engineering Geology 73(3), 247-265

Bonnard, C. and Noverraz, F. (2001). Influence of climate change on large landslides: Assessment of long-term movements and trends. International Conference on Landslides: Causes, Impacts and Countermeasures, 121-138

Brardinoni, F. and Church, M. (2004). Representing the landslide magnitude-frequency relation: Capilano River basin, British Columbia. Earth Surface Processes and Landforms 29(1), $115-124$
Chau, K.T., Sze, Y.L., Fung, M.K., Wong, W.Y., Fong, E.L. and Chan, L.C.P. (2004). Landslide hazard analysis for Hong Kong using landslide inventory and GIS. Computers \& Geosciences 30(4), 429-443

Coe, J.A., Kinner, D.A. and Godt, J.W. (2008). Initiation conditions for debris flows generated by runoff at Chalk Cliffs, central Colorado. Geomorphology 96(3), 270-297

Corominas, J. and Moya, J. (1999). Reconstructing recent landslide activity in relation to rainfall in the Llobregat river basin, Eastern Pyrenees, Spain. Geomorphology 30(1), 79-93

Dahal, R.K., Hasegawa, S., Masuda, T., and Yamanaka, M. (2006). Roadside slope failures in Nepal during torrential rainfall and their mitigation. Disaster mitigation of debris flow, slope failures and landslides, (Interpraevent 2007), Universal Academy Press, Tokyo, 2, 503-514

Dai, F.C. and Lee, C.F. (2001). Frequency-volume relation and prediction of rainfall-induced landslides. Engineering Geology 59(3), 253-266

Dai, F.C. and Lee, C.F. (2002). Landslide characteristics and slope instability modeling using GIS, Lantau Island, Hong Kong. Geomorphology 42(3), 213-228

DesInventar (2014). Inventory system of the effects of disasters. 29 de agosto 2014. http://www.desinventar.org/en/database

Glade, T. (1998). Establishing the frequency and magnitude of landslide-triggering rainstorm events in New Zealand. Environmental Geology 35(2-3), 160-174

Glade, T., Crozier, M. and Smith, P. (2000). Applying probability determination to refine landslide-triggering rainfall thresholds using an empirical "antecedent daily rainfall model". Pure and Applied Geophysics 157(6-8), 1059-1079

Gray, D.H. and Leiser, A.T. (1982). Biotechnical slope protection and erosion control. Van Nostrand Reinhold Company Inc.

Greenway, D.R. (1987). Vegetation and slope stability. In Slope stability: geotechnical engineering and geomorphology, edited by Anderson and KS

Guzzetti, F., Peruccacci, S., Rossi, M. and Stark, C.P. (2007). Rainfall thresholds for the initiation of landslides in central and southern Europe. Meteorology and Atmospheric Physics 98(34), 239-267 
Guzzetti, F., Peruccacci, S., Rossi, M. and Stark, C.P. (2008). The rainfall intensity-duration control of shallow landslides and debris flows: an update. Landslides 5(1), 3-17

IDEAM (2005). Estudio de la clasificación climática de Bogotá y cuenca alta del Río Tunjuelo. Instituto de Hidrología, Meteorología y Estudios Ambientales-IDEAM y Fondo de Prevención y Atención de Emergencias, 116 p.

Imaizumi, F., Sidle, R.C. and Kamei, R. (2008). Effects of forest harvesting on the occurrence of landslides and debris flows in steep terrain of central Japan. Earth Surface Processes and Landforms 33(6), 827-840

Iverson, R.M. (2000). Landslide triggering by rain infiltration. Water Resources Research 36(7):1897-1910

Iverson, R.M. and Denlinger, R.P. (2001). Flow of variably fluidized granular masses across three-dimensional terrain 1. Coulomb mixture theory. Journal of Geophysical Research 106(B1):537-552

Jiménez-Perálvarez, J.D., Irigaray, C., El Hamdouni, R. and Chacón, J. (2010). Landslide susceptibility mapping in a semiarid mountain environment: an example from the southern slopes of Sierra Nevada (Granada, Spain). Bulletin of Engineering Geology and the Environment 70(2), 265-277

Li, C., Ma, T., Zhu, X. and Li, W. (2011). The power-law relationship between landslide occurrence and rainfall level. Geomorphology 130(3), 221-229

Luna, B.Q., Remaître, A., van Asch, T.W., Malet, J.P. and van Westen, C.J. (2012). Analysis of debris flow behavior with a one dimensional run-out model incorporating entrainment. Engineering Geology 128, 63-75

Moreiras, S.M. (2005). Climatic effect of ENSO associated with landslide occurrence in the Central Andes, Mendoza Province, Argentina. Landslides 2(1), 53-59

Moya Barrios, J. y Rodríguez, J.A. (1987). El subsuelo de Bogotá y los problemas de cimentaciones. En: Memorias del VIII Congreso Panamericano de Mecánica de Suelos e Ingeniería de Fundaciones. Sociedad Colombiana de Geotecnia.

Moya Sánchez, J., Corominas Dulcet, J., Mavrouli, O.C. y Copons Llorens, R. (2013). Aproximación probabilística al número y tamaño de bloques en desprendimientos con fragmentación. VIII Simposio Nacional sobre Taludes y Laderas Inestables, Palma de Mallorca, 1107-1118
NOAA (2014). Changes to Oceanic Niño Index (ONI). http:// www.cpc.ncep.noaa.gov/products/analysis_monitoring/ensostuff/ensoyears.shtml (29 de agosto 2014)

Ouyang, C., He, S., Xu, Q., Luo, Y. and Zhang, W. (2013). A MacCormack-TVD finite difference method to simulate the mass flow in mountainous terrain with variable computational domain. Computers \& Geosciences 52, 1-10

Pradhan, B., Lee, S. and Buchroithner, M. (2010). A GIS-based back-propagation neural network model and its cross-application and validation for landslide susceptibility analyses. Computers, Environment and Urban Systems 34, 216-235

Pudasaini, S.P., Wang, Y. and Hutter, K. (2005). Modelling debris flows down general channels. Natural Hazards and Earth System Science 5(6), 799-819

Rickenmann, D., Laigle, D.M.B.W., McArdell, B.W. and Hübl, J. (2006). Comparison of 2D debris-flow simulation models with field events. Computational Geosciences 10(2), 241-264

Shrestha, B.B., Nakagawa, H., Kawaike, K. and Baba, Y. (2008). Numerical simulation on debris-flow deposition and erosion processes upstream of a check dam with experimental verification. Annual of the Disaster Prevention Research Institute 51(0), 613623

Suárez, J. (1998). Deslizamientos y estabilidad de taludes en zonas tropicales. Ed. Universidad Industrial de Santander, Bucaramanga, Colombia

Trauth, M.H., Bookhagen, B., Marwan, N. and Strecker, M.R. (2003). Multiple landslide clusters record Quaternary climate changes in the northwestern Argentine Andes. Palaeogeography, Palaeoclimatology, Palaeoecology 194(1), 109-121

Wang, C., Li, S. and Esaki, T. (2008). GIS-based two-dimensional numerical simulation of rainfall-induced debris flow. Natural Hazards and Earth System Science 8(1), 47-58

Wieczorek, G.F. (1996). Landslide triggering mechanisms. In Landslides: Investigation and Mitigation, eds. Turner and Shuster. Transportation Research Board - National Research Council, Special Report 247, 76-90

Zhou, C.H., Lee, C.F., Li, J. and Xu, Z.W. (2002). On the spatial relationship between landslides and causative factors on Lantau Island, Hong Kong. Geomorphology 43(3), 197-207 